Artículo de revisión

\title{
La cadena de producción del Cacao en Ecuador: Resiliencia en los diferentes actores de la producción
}

\author{
The Cacao production chain in Ecuador: Resilience in the different production actors

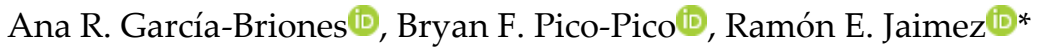 \\ Facultad de Ingeniería Agronómica, Universidad Técnica de Manabí, Manabí, Ecuador, 13132; agarcia7996@utm.edu.ec; \\ bpico4543@utm.edu.ec
}

*Correspondencia: ramon.jaimez@utm.edu.ec

\begin{abstract}
Citación: García-Briones, A., PicoPico, B. \& Jaimez, R., (2021). La cadena de producción del Cacao en Ecuador: Resiliencia en los diferentes actores de la producción. Novasinergia. $\quad 4(2) . \quad 152-172$. https://doi.org/10.37135/ns.01.08.10
\end{abstract}

Recibido: 20 mayo 2021

Aceptado: 06 agosto 2021

Publicado: 01 diciembre 2021

Novasinergia

ISSN: 2631-2654

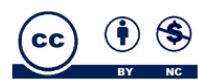

Copyright: 2021 derechos otorgados por los autores a Novasinergia. Este es un artículo de acceso abierto distribuido bajo los términos $\mathrm{y}$ condiciones de una licencia de Creative Commons Attribution (CC BY NC). (http://creativecommons.org/license s/by/4.0/).
Resumen: En los últimos tres años Ecuador, se ha situado como el tercer productor mundial de cacao. Varios factores como el cambio climático, altas concentraciones de cadmio y la pandemia global del COVID-19 están influyendo en la cadena productiva del cacao ecuatoriano. El análisis de las características de la cadena productiva y de comercialización del cacao, los datos de producción y exportación, las características de los tipos de cacaos, y productores muestran que Ecuador tiene una gran potencialidad para mantenerse en los estándares productivos. Aunque se han propuesto planes para la mejora competitiva del cacao ecuatoriano, estos planes no han considerado el entorno de producción del agricultor. $\mathrm{El}$ análisis de los factores que intervienen en la cadena del cacao ha permitido concluir que la resiliencia de la producción cacaotera ecuatoriana depende de la articulación de cinco acciones basadas en: (1) mejorar el entorno rural del productor, (2) fomentar la asociatividad y cooperativismo, (3) mejorar la productividad y el consumo del mercado nacional, (4) consolidar el mercado internacional, (5) e investigación orientada al mejoramiento. Estas propuestas se plantean como ejes de trabajo a mediano plazo. Es imperativo engranarlos en una acción encaminada a dar una mayor resiliencia a la cadena productiva.

Palabras clave: Cacao ecuatoriano, CCN 51, cacao fino de aroma, chocolate, comercialización cacao, cooperativismo, Theobroma cacao.

\begin{abstract}
Cocao is one of the traditional Ecuadorian export products. In the last three years, Ecuador has been the third-largest producer in the world. Several factors, such as climate change, high cadmium $(\mathrm{Cd})$ concentrations, and the global pandemic of COVID-19, are influencing the Ecuadorian cacao production chain. Based on an analysis of the characteristics of the Ecuadorian cocoa production and marketing chain, the production and export data, the features of the types of cocoas, the types of producers, it is shown that this country has great potential to remain within production standards. Although some previous plans have been proposed for the competitive improvement of Ecuadorian cacao, based on the production chain, these plans have not considered the farmer's production environment. The analysis of the different factors that intervene in the cacao chain has allowed concluding that the resilience of the Ecuadorian cacao production depends on the articulation of five actions based on: (1) improving the rural environment of the producer, (2) promoting associativity and cooperativity, (3) improve productivity and consumption in the national market, (4) consolidate the international market, (5) and research focused on improvement. These are proposals that are proposed as axes of work in the medium term. Therefore, it is imperative to engage them in action to provide further resilience to the production chain.
\end{abstract}

Keywords: Cacao marketing, CCN 51, chocolate, cooperativism, ecuadorian cacao, fine aroma cacao, Theobroma cacao. 


\section{Introducción}

El cacao es un árbol con tolerancia a la sombra, cultivado en el trópico húmedo, entre las latitudes $10^{\circ}$ Norte y $10^{\circ}$ Sur en los continentes Americano, Africano, Asiático y Oceanía (Muller \& Valle, 2012). cuya domesticación data de las culturas Mayas 200 años AC. y usado por los Toltecas y Aztecas en Centroamérica (Dreiss \& Greenhil, 2008). Los recientes hallazgos arqueológicos evidencian el uso del cacao hace 5300 años en el sitio de Santa Ana-La Florida en la provincia Zamora Chinchipe en el Sur-Este de Ecuador, parte superior del Amazonas (Zarrillo et al., 2018). La facilidad con la que el productor lo ha asociado con diferentes cultivos como plátano, frutales y árboles maderables (Franzen \& Borgerhoff, 2007; Jaimez et al., 2013), y debido a su tolerancia a condiciones de sombra permiten tenerlo integrado en sistemas de producción más diversos que brindan al productor otras alternativas de ingresos (Braga, Domene, \& Gandara, 2019).

Se ha estimado que la producción de cacao involucra entre 5 a 6 millones de agricultores a nivel mundial (Carr \& Lockwood, 2011). En el año 2019-2020, la producción mundial de cacao fue de 4,7 millones de toneladas. De esta producción anual, en Latino América se produjeron el $18.4 \%$ (0.9 millones t). Costa de Marfil y Ghana son los primeros dos países de mayor producción ( 2.1 y 0.8 millones $t$ respectivamente) y Ecuador el tercero (0.32 millones $\mathrm{t}$ ) (ICCO 2021). En enero del 2020 el precio por tonelada fue de 2603 USD. Los precios más bajos se registraron en el mes de julio del 2020 pagándose 2100 USD/t posteriormente comenzó el aumento hasta llegar a pagarse a 2407 US $\$ / t$ a finales de diciembre del mismo año.

Varios factores están influyendo en la producción de cacao y su comercialización a nivel global, entre ellos: el incremento paulatino de la temperatura del globo terráqueo, que está creando nuevos escenarios climáticos e influyendo en el cambio la dinámica poblacional de insectos y enfermedades, disminución de la disponibilidad de agua en el suelo en muchas regiones por aumento del período de sequía o también aumento en la probabilidad de eventos de exceso de lluvia con la consecuente inundación de terrenos (Márdero et al., 2012). Estos factores simultáneamente influyen negativamente para lograr una adecuada producción (Medina \& Laliberte, 2017).

Un segundo aspecto que en especial afecta a los países productores de Latinoamérica es la entrada en vigencia desde el 1 de enero del año 2019, de la regulación de contenidos de Cadmio (Cd) en los alimentos aprobada por la Unión Europea en el año 2014. La norma ha sido implementada debido a que la ingesta de chocolate contaminado con $\mathrm{Cd}$ ha aumentado paulatinamente y pudiera convertirse prontamente en un problema de salud pública (Maddela et al., 2020). Ello ha conducido a un mayor número de estudios con el objetivo de conocer los contenido de $\mathrm{Cd}$ en el suelo de las diferentes zonas cacaoteras de los países y los contenidos de $\mathrm{Cd}$ en la semilla de los diferentes genotipos que se siembran (Argüello et al., 2019; Barraza et al., 2019). A mediano plazo se persigue lograr a través de manejos de la plantación, y obtención de nuevos genotipos con menos absorción de $\mathrm{Cd}$, reducir los contenidos de $\mathrm{Cd}$ en las semillas de cacao, cuya regulación establece que los valores máximos deben ser $0.6 \mathrm{mg} / \mathrm{kg}$. 
El tercer factor es la pandemia del COVID-19 cuya propagación está afectando las cadenas de producción y suministro de alimentos. En un contexto global, debido a la crisis derivada de la pandemia del COVID-19, la cadena de producción y comercialización del cacao está sometido a varios factores que están creando escenarios que dificultan tanto la producción, su transporte, entrega, procesamiento y venta al consumidor final (Cadby 2020; Mena-Coronel \& GutiérrezJaramillo 2021).

En el mes de septiembre del 2020 en Ecuador se registró que las provincias de Pichincha y Guayas estaban entre las más afectadas debido al COVID-19 (Callejas et al., 2020). Esta última esta entre las tres provincias de mayor producción de cacao en Ecuador. Se puede esperar que la actual pandemia conlleve a cambios en las estrategias para la producción y la comercialización de este rubro. No obstante, la cadena del cacao en Ecuador no ha sido afectada y al contario se exportaron 60.000 t más que en el año calendario 2019 (Asociación de Exportadores de cacao, ANECACAO (2020). Es probable que el aumento se deba a un mayor número de extensiones sembradas en los últimos cinco años y que se están incorporado a la producción nacional. En el caso de Ecuador, el cultivo de cacao genera empleos para alrededor de 100000 familias, la mayoría en las zonas rurales (Barrera et al., 2019). En el año 2020 la producción de cacao generó ingresos cercanos a los 908 millones de USD de dólares (ANECACAO, 2020). Estas cifras muestran la importancia del cacao en el Ecuador en términos de generación de empleos, recursos financieros no derivados del petróleo, que además tiene un gran potencial de crecimiento. Por tanto, es imprescindible seguir evaluando como mantener y mejorar la producción cacaotera ecuatoriana implementando acciones que le permitan afianzarse y mitigar los efectos del cambio climático, estudiar estrategias para lograr disminuciones por contaminación de Cd y mantener la producción en los nuevos tiempos, que, sin duda alguna, está marcando la pandemia. Además, es de considerar que cambios en precios internacionales del cacao ecuatoriano, pueden depender de las medidas de comercialización del cacao africano que están cambiando y que pueden influir en la demanda de cacaos de países latinoamericanos (Parada-Gutiérrez \& Veloz-Cordero, 2021).

El presente trabajo tiene como objetivo realizar una revisión bibliográfica para analizar la producción de cacao en Ecuador para sistematizar una propuesta articulando acciones que conlleven a corto plazo a una mayor sustentabilidad e incrementar la resiliencia de la producción de cacao en Ecuador

\section{Metodología}

Se realizó una revisión de artículos relacionados con la producción y cadena productiva de cacao en Ecuador desde el año 2000. Para ello se utilizó la base de datos del Google Académico. Se consultaron las bases de datos de documentos técnicos del Instituto de Investigaciones Agropecuarias del Ecuador. La información relacionada a producción, ventas y exportaciones de diferentes productos de cacao fueron seleccionas de cifras de las bases de datos nacionales ecuatorianas que contenían información al menos desde 2017 obtenidas de las 
páginas del Instituto Nacional de Estadísticas y Censos de Ecuador (INEC), Banco Central del Ecuador y de la Asociación Nacional de Exportadores de Cacao (ANECACO). Documentos técnicos de grupos de talleres de trabajo en relación con el cacao ecuatoriano también fueron revisados desde 2010. Se seleccionaron 36 artículos científicos originales que sustentaban información relacionada a la producción y la cadena del cacao en Ecuador. La revisión conllevó a identificar las áreas en las que se deben emprender actividades que conlleven a una mayor sustentabilidad y resiliencia de la cacao-cultura ecuatoriana.

\section{Resultados}

\subsection{La producción de cacao en Ecuador: Características}

Tipos de cacao y productores

En Ecuador se cultivan dos tipos de cacao: los cultivares tipo Nacional, y el clon CCN 51. Los primeros cultivados en su mayoría en sistemas agroforestales por pequeños productores (Melo \& Hollander, 2013) y algunas empresas que tienen extensiones que sobrepasas las 120 ha. La calidad sensorial del cacao tipo Nacional es reconocida mundialmente y por el cual se paga precios altos en los mercados internacionales. También hay cacaos llamados complejos híbridos de cacaos nacionales con cacao trinitarios (Ortiz et al., 2019). Existe una alta diversidad genética de los nacionales nativos y está relacionado a la introducción de cacaos de otros países y sus cruces (Carranza et al., 2020) El CCN 51 es un clon obtenido por Homero Castro a partir de los cruces (ICS95 x IMC 67) x Oriente 1 en su finca Theobroma en la región Naranjal, Provincia del Guayas. Es cultivado desde 1980 comercialmente, de mayor productividad, pero de menor calidad que los cacaos nacionales. Se ha cultivado como un monocultivo y en la mayor parte de los casos sin sombra.

Debido a que en el país no se ha planteado una diferenciación de precios entre ambos tipos, se ha propiciado que Ecuador venda la mezcla de ambos ocasionando la pérdida de la calidad y generando desconfianza en los compradores internacionales (Melo \& Hollander, 2013; DíazMontenegro, Varela, \& Gil, 2018; Salgado, Morillo, \& Ulloa, 2019). La ICCO penalizó esta disminución de calidad con una reducción del $25 \%$, es decir en la totalidad del cacao que se exporta $75 \%$ es cacao fino de aroma (Troya, 2013).

Existen estudios que han diferenciado grupos de productores de cacao en función de estrategias de medios de vida. Por ejemplo, Díaz-Montenegro et al. (2018), en la provincia de Guayas estudiando 188 productores, identificó cuatro tipos de productores basándose en la proporción de tierras de sus fincas dedicadas a cacaos nacionales y al CCN 51, además del tipo de mano de obra utilizada en las diferentes labores. Estos cuatro grupos se asocian en dos grupos, los primeros dependientes en la intensificación agrícola dedicada al cacao con diferenciación en la cantidad de terreno dedicado al cacao nacional y al CCN 51 y dependen fundamentalmente de la mano de obra familiar y los otros dos grupos diversifican sus aportes financieros con una mayor cantidad de cultivos en la finca y entradas financiera al núcleo 
familiar en actividades no agrícolas con empleos fijos o transitorios. Otra clasificación realizada por Barrera et al. (2019) con otros parámetros evaluada a través de encuestas realizada a 386 productores de Manabí, agrupa a los productores en tres grupos: el primero representa el $37 \%$ y son hogares con pequeñas fincas cercanos a 3 ha, en su mayoría no tienen riego y venden su cacao a minoristas o asociaciones. El segundo grupo que son el $54 \%$ venden sus productos a minoristas y la extensión de sus cultivos es de 4 ha, cerca del $25 \%$ dispone de riego. El tercer grupo que representan el $8.3 \%$ restante tiene extensiones de cacao promedio de 4.6 ha, menos del $10 \%$ tiene riego, mantienen prácticas de manejo y venden su cacao a bodegas mayoristas (Barrera et al., 2019). Llama la atención en este estudio, que un alto porcentaje no tengan riego.

Entre los productores de cacao nacional hay una distinción clara de dos grupos: los pequeños productores, con menos de 10 ha con sistemas de producción limitado, con plantaciones asociadas a otros cultivos y árboles y el grupo de los medianos y grandes productores cuyas plantaciones en su mayoría son monocultivos. Los productores de CCN 51 tienen plantaciones comerciales que comenzaron a fundarse desde hace 25 años, muchas de ellas con riego y manejados con maquinaria y diseños de plantación diferentes a las utilizadas en los sistemas convencionales e inversiones altas en manejo de enfermedades y plagas. Igualmente tiene un buen manejo de postcosecha (fermentación y secado) y manejan certificaciones internacionales como UTZ y Rainforest, además de generar una cartera de empleos tanto directo como indirectos (Henry, Morillo, Salgado, \& Ulloa, 2018). Existe también en el país pequeños productores con plantaciones de CCN 51 y algunos presentan un mosaico de cacaos nacionales y CCN 51.

El cacao en Ecuador se produce en 16 de las 24 provincias, en las cuales está dividido Ecuador para un total de 590579 ha plantadas y 527327 ha cosechadas (INEC, 2020). Los productores pequeños de cacao nacional se reúnen en asociaciones en las diferentes provincias (Tabla 1). Esmeraldas es la provincia con el mayor número de asociaciones (51), seguido por la provincia de Manabí con 28. Provincias como Morona, Pastaza, Cañar tienen una sola asociación. En las fincas de pequeños productores se pueden encontrar cacaos de tipo Nacional, complejos de Nacional-trinitario y CCN 51. Se distinguen pocos productores clasificados como medianos o gran productor. Existe un grupo de productores de CCN 51 asociados en APROCAFA, así como otro productores con superficie de más de 100 ha de CCN 51 produciendo en forma independiente (Henry et al., 2018). Es de resaltar que, entre las provincias de Los Ríos, Manabí Guayas suman el $61.3 \%$ de la superficie total plantada (INEC, 2020).

Los estudios mencionados anteriormente y los datos de superficies de producción en los productores muestran la diversidad de pequeños productores que se pueden encontrar cuyas variaciones puede ser debidas al tipo de cacao y la extensión de terreno usado para cada uno, mano de obra que usan, comprador final, riego disponible y diversificación de cultivos. Actualmente las nuevas plantaciones de cacao ecuatoriano son de CCN 51 debido a su alta producción y debería ser analizadas con detalle ya que pudieran indicar un mayor desplazamiento de cacao nacional que comenzó con la entrada de cacao trinitarios y que actualmente ocurre con el CCN 51. Por ejemplo, nuevos clones tipos nacionales(EETP 800, 
EETP 801) de alta producción que han sido obtenidos, donde uno de sus padres es el CCN 51, fueron liberados hace tres años y están comenzado a usarse en varias localidades del país (LoorSolórzano et al., 2019).

Tabla 1: Distribución del número de asociaciones* de pequeños productores de cacao nacional y CCN 51 en las provincias de Ecuador Henry et al. (2018).

\begin{tabular}{cccc}
$\begin{array}{c}\text { Cacao Nacional } \\
\text { medianos y } \\
\text { grandes }\end{array}$ & \multicolumn{2}{c}{ Cacao Nacional y CCN 51 } & CCN 51 grandes \\
\hline Organizaciones pequeñas de productores & \\
\hline 10-20 productores & Esmeraldas (51) & Napo (15) & No asociado (1) \\
& Orellana (5) & Morona (1) & APROCAFA (6 \\
& Pastaza (1) & Cotopaxi (2) & productores) \\
Cañar (1) & Zamora (2) & \\
Bolívar (4) & Santo Domingo (10) & \\
& Pichincha (13) & Manabí (28) & \\
El Oro (3) & Guayas (24) & \\
& Los Ríos & Santa Elena & \\
\end{tabular}

*Entre paréntesis número de asociaciones

En la postcosecha el secado es una actividad realizada por la mayoría de los productores en sus propias fincas, variando el método e infraestructura donde la realizan. Por ejemplo, Barrera et al. (2019) encontraron en la provincia de Manabí que un porcentaje muy bajo de productores (3 $\%$ ) seca en el asfalto de carretera, y los mayores porcentaje usan tendal de cemento (38\%) y madera (34 \%). Hay un bajo porcentaje $(7.5 \%)$ entre los productores de secar las almendras dentro de estructuras bajo cubierta tipo marquesinas. Es de hacer notar que los productores no almacenan el cacao, al contrario, lo venden inmediatamente.

Dinámica de la exportación

En la dinámica anual de exportación de cacao en Ecuador, la mayor cantidad de exportación se centra entre los meses de octubre a diciembre que coincide para algunos lugares de la región costera con el comienzo de la época de lluvia. En estos meses la exportación en el periodo 2016-2018 estuvo entre 36000 y $48000 \mathrm{t}$ mensual (Figura 1). Ya para los meses de enero y febrero la exportación baja $50 \%$ a valores cercanos a las 18000 t mensuales. El período entre mayo - julio es el de menor exportación con un promedio aproximado de 16500 t y coincide con los primeros tres meses del periodo de sequía en la zona de la costa ecuatoriana que dura alrededor de 6 meses. Esto quiere decir, que el crecimiento y llenado de mazorca ocurre en el momento de mayor limitación hídrica en plantaciones que carecen de riego, en especial en la región de la costa ecuatoriana.

En los datos estadísticos de ANECACAO se muestra que las exportaciones de cacao en el 2014 fueron de 234277 t. En el 2015 llegó a 260540, en el 2016 se alcanzó los 249632, en el 2017 aumentó a 301526 y en el 2018 se obtuvo 315571 t y para el año 2020 se reportaron $360000 \mathrm{t}$ (ANECACAO, 2020). El evidente crecimiento en la exportación de almendras de cacao, al parecer es debido en parte al crecimiento de superficie sembrada que ha llevado al 
rejuvenecimiento de la edad promedio de las plantaciones (ahora más productivas que las plantaciones antiguas).

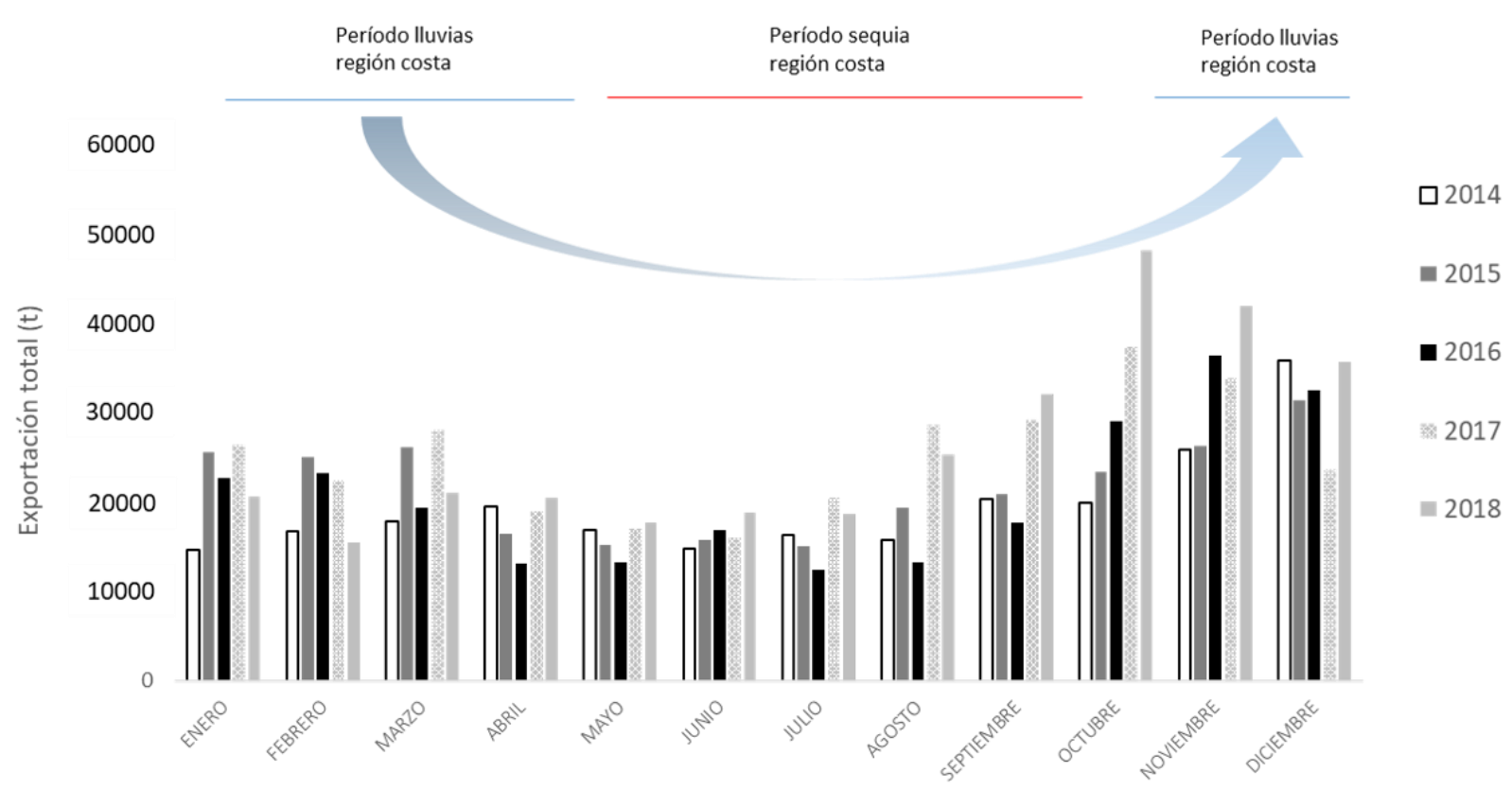

Figura 1: Exportaciones totales mensuales de cacao de Ecuador entre los años 2014-2018 (ANECACAO, 2020).

Como se ha planteado los productores en Ecuador están diferenciados en el tamaño de sus fincas, y se estima un incremento de al menos un $40 \%$ en el número de productores. Por ejemplo, se conoce que el 2007 existían más 100 mil productores y para el año 2018 se contabilizaban 189000 productores, donde más el $84 \%$ solo poseían fincas de menos de 5 ha (INEC, 2018), lo cual evidencia que la gran parte de la producción del cacao proviene de pequeñas fincas. Solo cerca de un $15 \%$ cultivan hasta 20 ha y el $1 \%$ restante catalogado como medianos y grandes productores posee fincas por arriba de 20 ha (INEC, 2020). Para el año 2018 se estima alrededor de 573000 ha sembradas de cacao de las cuales 248000, 203000, 32500 y 17000 ha corresponden a pequeños productores $(<5 \mathrm{ha}$ ), pequeños productores (entre 5 y 20 ha), mediano productor (entre 20 y $50 \mathrm{ha}$ ) y productor grande (> $50 \mathrm{ha}$ ) (INEC, 2018).

El promedio de edad en los productores esta alrededor de los 57 años (Barrera et al., 2019; INEC, 2018). Muchos de los jóvenes pertenecientes a estos hogares se han ido a las ciudades. Sin embargo, recientemente la reducción de empleos ha obligado la vuelta al hogar. Es decir, es probable que este sea el momento, para comenzar con proyectos de formación técnica en el manejo del cacao. El $70 \%$ de los productores cacaoteros tienen solo 8 años de educación primaria (Barrera et al. 2019; INEC, 2018) por tanto, idear un programa de capacitación técnico y extensivo sentaría mejores bases técnicas para el manejo del cacao en todas las regiones cacaoteras del país. Es indudable que se debe dar una mayor formación en el manejo de riego, así como estrategias de fertilización, podas y control fitosanitario. 


\section{Comercialización}

Existen dos caminos en la comercialización del cacao en Ecuador: El cacao que va pasando por una cadena de intermediarios hasta llegar al exportador que denominamos el mercado interno y el segundo camino es el que se vende directamente al exterior, actividad que la realizan muy pocas empresas. La comercialización externa, se fundamenta en la exportación de granos en dos variedades; El tipo Nacional y el clon CCN 51; La forma como se exportaba el cacao producido en Ecuador en el año 2017 era: 87 \% en grano, 12 \% semielaborados y un 0.8 \% correspondió a productos terminados (Morales et al., 2018; Henry et al., 2018). Los diferentes eslabones de la cadena básicamente se dedican al manejo del grano y su comercialización y es lo que se exporta en un mayor porcentaje. Pocas iniciativas de procesamiento hay en el país, lo cual lo caracteriza como una cadena no generadora de valor agregado.

La figura 2 muestra la cadena en forma general de la comercialización de cacao. En el entorno rural, caracterizado por pocos servicios públicos de asistencia médica y carreteras con poca accesibilidad, la compra de semilla puede ser realizada por intermediarios pequeños que visitan las fincas que según Barrera et al. (2019) es el sector que capta más producción, para luego venderla a compañías. Los intermediarios recorren las fincas para comprar directamente el producto, que por lo general se compra en baba y en algunas regiones como Manabí se vende seco. Otra manera de comercializar el cacao por parte de los productores es efectuando la venta a los acopiadores rurales de la zona, que reúnen lotes más amplios y a su vez lo venden a los mayoristas para que eventualmente sea entregado a los exportadores. Una variante es también cuando el agricultor va a los centros poblados más cercanos a vender su producto a intermediarios, quienes acopian el cacao en lotes más grandes para entregarlo al exportador. Una tercera forma, es cuando el agricultor, generalmente el que tiene una propiedad más grande, vende su producto directamente al exportador (Morales et al., 2018). En algunas regiones existen cooperativas o asociaciones de productores donde el precio de compra es mayor además de recibir algunos otros beneficios por la asociación.

Un ejemplo que muestra en términos porcentuales el manejo de la cadena de comercialización se evidencia en la provincia de Los Ríos, en donde el $76.9 \%$ del cacao se vende al comerciante intermediario (de estos, el $55 \%$ al intermediario que compra en la finca y el $45 \%$ al intermediario del poblado más cercano), el $11.5 \%$ lo vende directamente al exportador y el restante $11.5 \%$ entrega su cacao a asociaciones que se encargan de comercializar directamente. En términos de aproximación en Ecuador el $70 \%$ de los productores entregan su cosecha a los intermediarios, quienes finalmente venden a los exportadores (Morales et al., 2018). No hay diferencia de precios entre los cacaos tipo nacional y CCN 51. Se debería evaluar el establecimiento de un diferencial de precios por la calidad de cacao. Esta característica de igual precio de compra por cacao Nacional y CCN 51 produce una menor rentabilidad al productor del cacao Nacional debido al menor rendimiento de los Nacionales y además no reconoce la calidad sensorial y una prima que se recibe en el mercado internacional (Barrezueta-Unda, Prado, \& Sarmiento, 2017). 
La otra parte de cadena lo constituyen, las grandes empresas que tienen plantaciones que pueden estar basadas en clones nacionales o en el clon CCN 51. Son monocultivos extensos (mayor a 80 ha), con sistemas de fertirriego, con programas de controles fitosanitarios y uso de maquinaria para labores de poda. Entre estas empresas algunas venden a exportadores internos nacionales o directamente comercializan con compradores de compañías externas y otras exportan directamente su producto (García, Montaño \& Montoya, 2012).

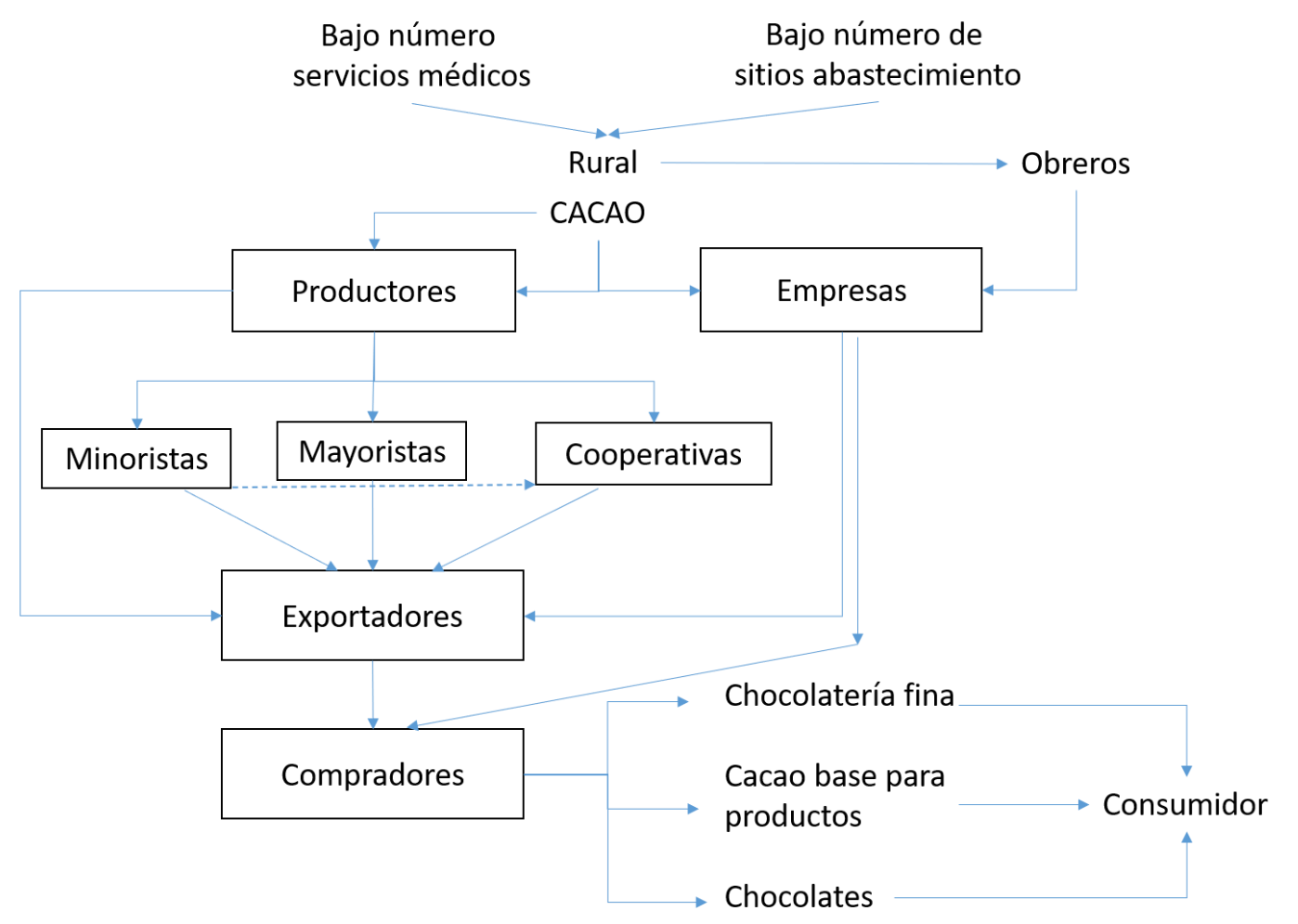

Figura 2: Cadena de producción y comercialización de cacao en Ecuador.

Existe una industria de semielaborados produciendo pasta, licor, manteca y polvo dominado por grandes empresas, cuyas producciones van al mercado externo. La producción de chocolates nacional es conformada fundamentalmente por pequeñas empresas que elabora sus productos tanto para el mercado nacional como para el internacional (CEPAL, 2013; Henry et al., 2018 ). En el caso de Ecuador los productos y bienes que se derivan del cacao (manteca, aceites y licor) se emplean como materias primas en la industria de chocolates y en la industria de cosméticos (labiales, cremas y lociones) y farmacéutica (Barrezueta-Unda, Blacio, \& Abad, 2018).

Por otra parte, el precio internacional de la bolsa de nueva York rige el precio nacional. La cantidad de dinero recibida por la exportación del cacao va disminuyendo en la cadena, de manera que el productor recibe la menor participación (Vasallao, 2017) que es alrededor del 75 $\%$ del precio de exportación (Barrera et al., 2019). Se ha planteado que los gastos de traslado, presentación y comercialización son primas que van quedando en cada eslabón de la cadena, no tomándose en cuenta los riesgos del productor. Estos incluyen enfermedades y plagas de las plantas, eventos en las condiciones ambientales (largo periodo de sequía o inundaciones), 
sumado a las condiciones de servicios (transporte, luz, caminos de acceso, entre otros) que en zonas alejadas no son de buena calidad y no ofrecen un buen servicio al productor. Sumado a esto se presenta los bajos rendimientos que están entre 400-500 kg/ha (Ibarra, 2019).

Según ANECACAO en el período entre el año 2018-2020, el país que compró la mayor cantidad de cacao ecuatoriano fue Indonesia seguido de Estado Unidos, luego Malasia, y Holanda, México el quinto y Alemania el sexto. Estos 6 países han comprado el $78 \%$ de la producción total de estos tres años (978000 t). Indonesia y Estados Unidos han comprado el 4.3 \% (21.6 y 20.7, respectivamente) con tendencia en los dos últimos años a una mayor compra, mientras que Holanda disminuyó su compra en un $41 \%$ en el 2020 (figura 3). Es de resaltar que el continente europeo ha comprado aproximadamente el $20 \%$ en los últimos cuatro años.

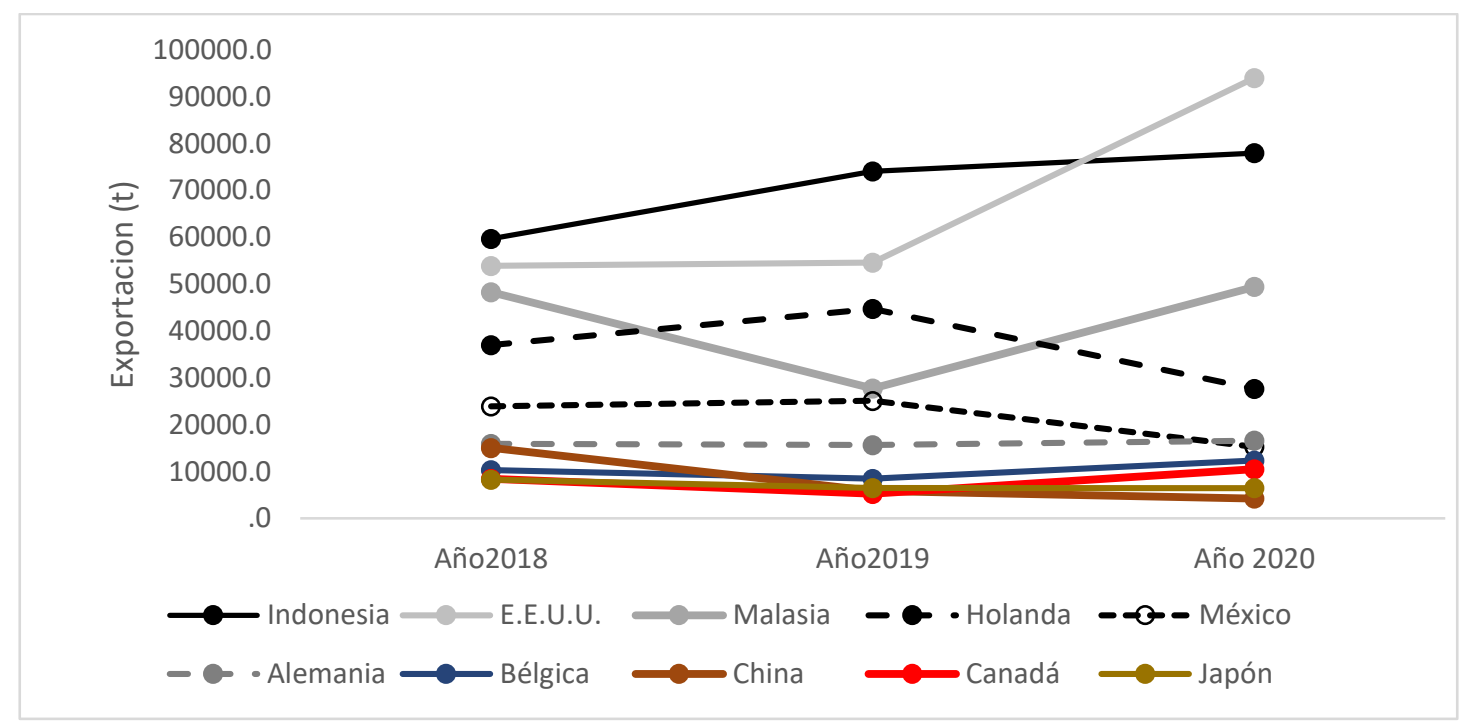

Figura 3: Países de destino de las exportaciones de cacao en grano de Ecuador (ANECACAO, 2021).

Plan de mejora competitiva para el desarrollo agroindustrial del cacao

Existe un documento reciente donde se plantean las líneas estratégicas y las actividades a realizar para implementar un desarrollo del cacao en los próximos 5 años (Salgado et al., 2019). En dicho plan se contempla las actividades a desarrollar con el objetivo de lograr que Ecuador sea referente del cacao por la calidad y lograr el reconocimiento del precio basado en sus perfiles de sensoriales sostenibles y trazables. La meta es llegar al año 2025 a $500000 \mathrm{t}$ de exportación de los cuales 400 mil t sean de cacao en grano, 80 mil t de semielaborado y $20 \mathrm{mil} t$ en chocolate. Es de destacar que se ha planteado como objetivo lograr que las metas de exportación sean de 200 mil t como cacao en grano sea tipo Premium con un perfil aromático definido y las otras 200 mil t estén representadas por cacao en grano con certificación de sostenibilidad.

Este plan se enmarca en seis líneas estratégicas: 1) Recuperar la confianza de los compradores en el cacao ecuatoriano: Acción que requiere establecer un sistema de calidad y certificación nacional basándose en la trazabilidad y tener una identificación organoléptica nacional. La amplia diversidad de genotipos es una fortaleza importante para lograr tener una oferta diversa de 
perfiles aromáticos. 2) Fomentar y fortalecer la asociatividad: Actividad fundamental para lograr una mayor integración de los productores, mejor capacidad para lograr certificaciones y tener una mayor representatividad a nivel nacional en el ámbito social y político en la toma de decisiones nacionales hacia el cultivo. 3) Reducir costos de producción y mejorar la productividad y el manejo tecnológico del cultivo: Se plantea tener 45000 ha de cacao nacional renovadas, $60000 \mathrm{y}$ 15000 ha de cacao CCN 51 y Nacional respectivamente, rehabilitadas. Además, se platea implementar un sistema nacional de asistencia técnica en la cual participe el sector público y privado.4) Incentivar la inversión nacional e internacional: Basándose en una estrategia que atraiga inversiones para lograr incrementar la industria de semielaborados y elaborados. Se plantea identificar y establecer complejos productivos para el desarrollo agroindustrial y facilitar la incorporación de nuevas empresas y negocios en la cadena agroindustrial. La tarea es lograr recursos para la investigación e innovación desarrollo y transferencias de tecnologías aplicadas al valor agregado y la creación de un centro nacional de investigación y desarrollo en cacao. 5) Establecer una institucionalidad funcional: Fundamentalmente se ha plateado reestructurar el consejo consultivo del cacao y lograr la reactivación de 14 mesas provinciales para articular y mantener el plan mejora competitiva del cacao. 6) Posicionar el cacao ecuatoriano: A través de estrategia de divulgación, comercialización se ha planteado aumentar el consumo de cacao en el país. Simultáneamente es preciso mantener la presencia en ferias y eventos internacionales promocionando al cacao ecuatoriano, además de estructurar un buen proyecto que promociones el cacao cultura ecuatoriana sus éxitos y la calidad de sus chocolates. Existe el decreto ejecutivo nacional Nro. 791 del 17 de junio del año 2019 en la cual el Programa de mejora competitiva del cacao se eleva a política pública y las entidades de la función ejecutiva están facultadas para ejecutar acciones para fortalecer y promover la cadena agroindustrial del cacao y sus derivados

\section{Discusión}

\subsection{Propuesta de ejes de acción para lograr mayor resiliencia en la Cadena productiva de cacao}

En base la revisión realizada se ha identificado que la producción de cacao en Ecuador está basada en un gran número de pequeños productores (80 \%), no asociados en su mayoría, con un porcentaje bajo con sistemas de riego y una producción nacional que vende el $80 \%$ al exterior en forma de grano. Además, el plan de mejora competitiva propuesto recientemente no considera el entorno en el que se desenvuelve el productor. Con la finalidad de fortalecer la cadena productiva se proponen 5 ejes de acción (figura 4) que conllevarían a una mayor resiliencia del sector cacaotero. Se parte de la mirada inicial a los pequeños productores y su entorno rural, su organización y mejora de la productividad, combinado con el cambio en la estrategia de mejora de la calidad y trazabilidad para dar una nueva visión a nivel internacional del cacao ecuatoriano. Es fundamental dar una mayor relevancia a la investigación que debe ser el pilar para la construcción de una tecnología nacional. Estos ejes deben interrelacionarse y realizarse coordinadamente. A continuación, se explican. 


\section{ACTIVIDADES}
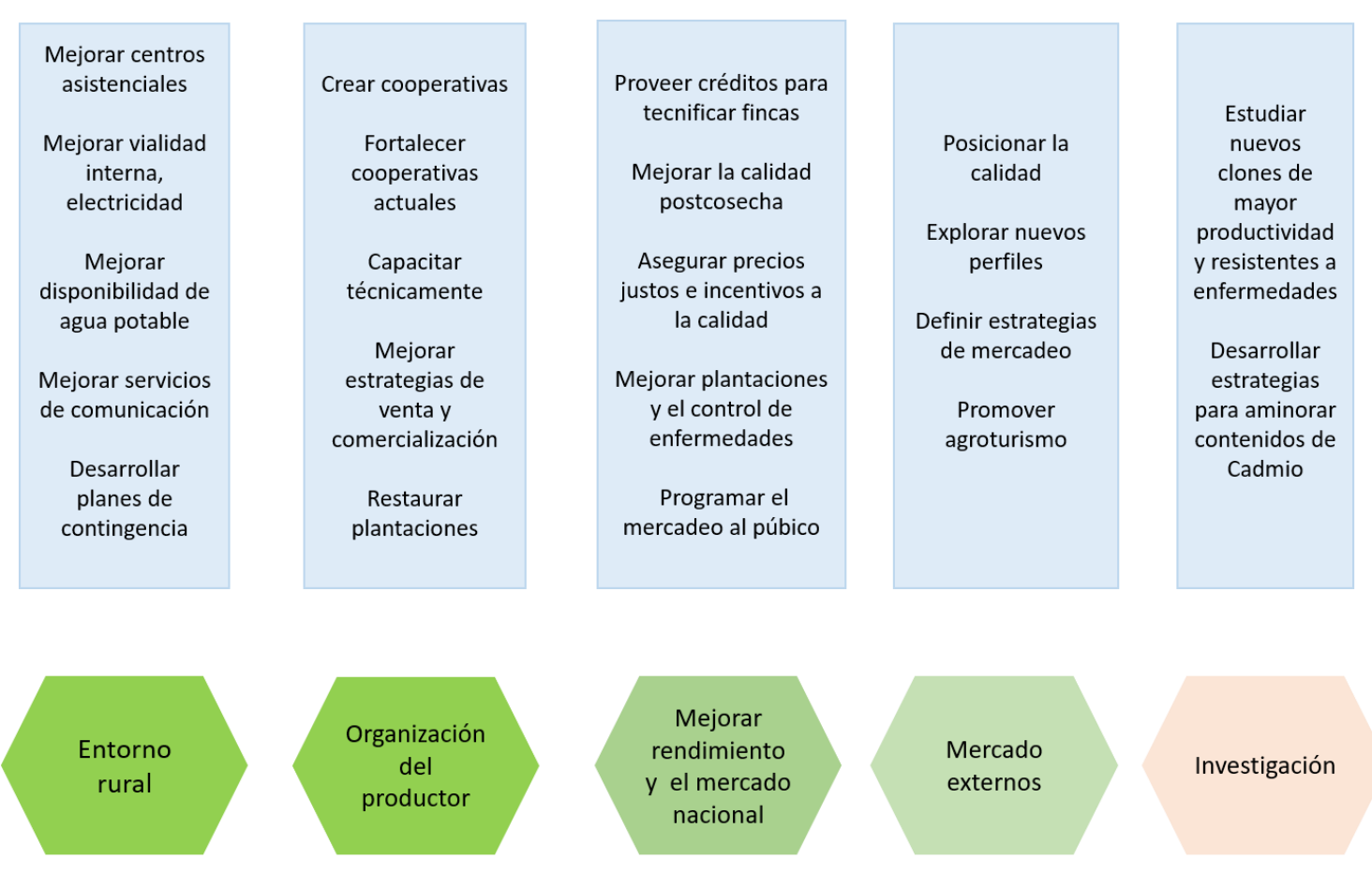

Ejes de Acción

Figura 4: Ejes de acción para fortalecer la resiliencia, aumentar la calidad y la producción de cacao ecuatoriano, así como actividades que se deben emprender o continuar realizándose para cada eje.

Mejora del entorno rural del productor.

La mirada al entorno en el que vive el productor nos lleva a preguntarnos sobre su calidad de vida y que hacer para que sea más confortable. En el sector rural, la tercera parte de los productores cacaoteros (63000) poseen seguro campesino, es decir hay alrededor de 120000 trabajadores sin seguros de salud (INEC, 2018), ni sistemas de amparo social por desempleo y sus ingresos para la manutención dependen del trabajo diario. Esto implica considerar lo extremadamente frágil y vulnerable que pueden ser los productores ante aumentos de contagios en las zonas por el SARS-COV-2 y otras enfermedades. Tal fragilidad es aumentada por la escasez o lejanía de instituciones de salud. El SARS-COV-2 ha conllevado a la saturación de los centros de salud debido a su rápida infección con una alta incidencia en personas de edad avanzada y condiciones médicas preexistentes (Callejas et al., 2020). Es claro la prioridad de proteger las vidas de los miles de productores que se enfrentan a importantes riesgos económicos y de salud como resultado de la crisis generada por la pandemia.

Se requiere un plan prioritario que debiera establecerse como un trabajo continuo a fin de lograr mejorar los servicios básicos de agua, electricidad y comunicación y de esta manera mejorar las condiciones de vida del productor en el campo. Actualmente, disponer de eficientes sistemas de internet es básico como medio para lograr divulgar información y apoyar la educación de niños y jóvenes. Aproximadamente el 21 \% de los estudiantes de Ecuador en la educación 
básico y bachillerato unificado se encuentra en la zonas rurales y la educación a través de internet a aumentado la brecha de desigualdad en la formación de niños y jóvenes (VivancoSaraguro, 2020). Otro aspecto importante es mejorar la vialidad interna y la accesibilidad a las fincas. En periodos de lluvia la movilización interna es difícil.

Se necesita conocer las limitaciones que tienen los agricultores, tales como acceso limitado a la infraestructura de producción de alta calidad, por ejemplo, acceso limitado a insumos, capital humano de asesoramiento y capital financiero. Existe una realidad la cual pudiera convertirse en una limitante: Los propietarios de fincas cacaoteras en un $70 \%$ son personas cercanas a los 60 años y la generación de relevo está faltando. Es propicio el momento para incentivar el retorno de muchos jóvenes al campo, pero ofreciendo mejores servicios que den mejores condiciones de calidad vida rural.

Es importante evaluar y priorizar la seguridad alimentaria del pequeño productor, que puede deteriorarse, dependiendo de las medidas que el país establezca para evitar una alta propagación de COVID-19. Estos aspectos muestran, como el productor, el primer eslabón de la cadena merece especial atención, debido a la vulnerabilidad que presenta y lo imprescindible de mejorar su entorno de servicios que conlleven a mejorar su calidad de vida.

Fomentar el cooperativismo (mayor cantidad de cooperativas de productores): generar más competencia

Coincidiendo con el plan de mejoramiento mencionado, en estos momentos es importante la organización de las cooperativas y pareciera que son las organizaciones que pueden dar un mayor apoyo a los productores debido a que sus integrantes son de la misma localidad y poseen un fin común que es la venta de su producción. Se conoce que para el año 2019 cerca de las dos terceras partes de los productores que existen en cacao no pertenecen a ningún tipo de asociación. Esto puede indicar una poca disposición de los productores para organizarse en cooperativas, lo cual debiera estudiarse para conocer las razones.

Las cooperativas pueden ser un mecanismo de organización que a mediano plazo permita que los productores consoliden un sistema de servicios creados por ellos mismos que los apoyen en sus diferentes actividades. Son estructuras económicas importantes en muchas comunidades rurales y son formas de capital social, organizadas para brindar servicios a sus miembros. Un aspecto importante y función que debe estar arraigada en las cooperativas, son las actividades de capacitación de sus miembros, a través de técnicos y profesionales que tengan una sólida formación en el manejo del cultivo, a fin de manejar las fincas de los agremiados bajo conceptos técnicos que permitan mejorar la producción con estrategias de sostenibilidad. Barrera et al. (2019) encuentran que $58 \%$ de los productores manifiesta la importancia de las cooperativas en su rol para el apoyo técnico y el entrenamiento. El concepto de cooperativismo debe manejare como una empresa con miras a mediano plazo de expansión tanto en el número de asociados como en su capital de inversión.

Mejorar la productividad y el mercado nacional

En esta línea, varias actividades deben realizarse simultáneamente. 
Un objetivo colectivo es lograr la transformación de las fincas en negocios modernos que permitan a los agricultores obtener ingresos suficientes para lograr un mejor nivel de vida. Esto implica apoyar en especial a los pequeños productores con financiamientos para rehabilitar las plantaciones viejas con siembras de clones altamente productivos adaptadas a las zonas y condiciones, acompañadas de una mayor tecnificación, lo cual incluye planes piloto como: podas, controles eficientes y sustentables de plagas y enfermedades, sistemas de riego, incluir en las plantaciones los nuevos genotipos existentes y fomentar la disponibilidad de plántulas certificadas. Es prioritario llevar el riego a las fincas de los pequeños productores. La falta de una adecuada disponibilidad de agua en el suelo es probablemente una de las mayores limitantes para aumentar los rendimientos nacionales.

El fortalecimiento de jardines clonales y material de siembra de calidad es una tarea importante para realizar. Es imprescindible la oferta de plántulas certificadas asegurando por parte de las asociaciones y viveros comerciales la veracidad de los genotipos comercializadas. Actualmente se cuentan con genotipos comerciales entre ellos genotipos INIAP (EET 103, EET 96, EET 95, EET 575, EET 576, EET 544, EET 558, EETP 800, EETP 801) CCN 51, JHVH-10, PMA 12, Sacha Gold y Sacha (Super arboles) y Martines 02. La estrategia es fomentar la producción de cacaos nacionales lo que afianzaría la calidad del cacao fino ecuatoriano internacionalmente.

Mejorar el manejo de los sistemas agroforestales y diversificar es también un punto importante y la oferta de otras especies entre ellas: palo prieto (Erythrina glauca), guabas (Inga sp), guayacán (Tabebuia achrisanta) y frutales Zapote (Matisia cordata) Limón (Citrus limón) (Coello \& Haro, 2012) debe implementarse. Estas actividades debieran realizarse a través de las cooperativas de productores con el apoyo y la participación de la industria en estrecha colaboración con los gobiernos de origen. La estrategia de la diversificación de la producción en pequeños productores amortiguaría cambios a la baja en los precios de comercialización.

b) Precio en función de la calidad

Un problema fundamental en la cadena de producción de cacao ecuatoriano es la no generación de un precio oficial nacional entre el cacao nacional y el CCN 51 ((Barrezueta-Unda, Prado, \& Sarmiento, 2017). Una de las prácticas de la calificación que realiza el comerciante al producto consiste en la apreciación visual, estimar el grado de fermentación, así como el porcentaje de humedad, igualmente, la calidad se mide por la mezcla de variedades CCN 51 y Cacao tipo Nacional, la contaminación con elementos químicos o pesticidas que son restringidos en los mercados internacionales que prefieren optar por prácticas orgánicas. Estos parámetros normalmente establecen amonestaciones, que influyen en el descuento del precio final del cacao (Morales et al., 2018). Es preciso trabajar con el productor y hacerle conocer las penalizaciones establecidas y que influyen en el precio del cacao. Esto conlleva a seguir planteando estrategias para que pequeños productores adquieran información y capacitación 
en las técnicas de fermentación y secado. A la par deben desarrollarse nichos de cacaos premium donde probablemente el precio dependerá de negociaciones específicas.

Por otra parte, es importante el fomento del consumo de chocolates y productos de calidad derivados del cacao y elaborados en Ecuador. Es prioritario la difusión de los beneficios del consumo de chocolate de calidad y de alta pureza y un bajo contenidos de leche y azúcar. La estrategia es formar una conciencia nacional de consumo que se vaya arraigando en el país. Para ello se debe implementar estrategias de divulgación usando diferentes medios de difusión, tanto de radios, periódicos, redes sociales. También implica por parte de empresas la inversión en mercadeo

\section{Mercado Internacional}

Se cuenta con alta producción, calidad sensorial que ofertar e indiscutiblemente la tarea importante, es orientar más acciones a sentar mejores bases que proyecten el cacao ecuatoriano a nivel internacional. Como lo planea el plan de mejoramiento es tarea del Ecuador recuperar su prestigio en base a un sistema interno nacional de trazabilidad y una buena oferta de perfiles garantizados. Es importante tener presente que los mercados internacionales y consumidores está queriendo que los productos tengan certificaciones orgánicas, certificado de ausencias de pesticidas comercio justo, lo cual da mayor valor (Henry et al., 2018). Esto implica un plan estratégico de oferta, de mostrar las actividades exitosas del Ecuador para recuperar la confianza de los compradores. La demanda del tipo de cacao lo establece el mercado internacional y es prioritario establecer estrategias de oferta en función de lo que es requerido por los compradores.

Probablemente la inversión en agroturismo se debe fortalecer. La oferta de visitar fincas y productores es una estrategia para incursionar e incluso la oferta a jóvenes de otros países que deseen realizar pasantías en fincas es una actividad que promueve un turismo diferente.

Igualmente, es importante evaluar las posibilidades de entrada del cacao ecuatoriano en el continente europeo en un mayor porcentaje. Solo el $20 \%$ del cacao ecuatoriano es comprado por países europeos. Esta realidad obliga a reevaluar las estrategias de mercadeos y ofertar un cacao de alta calidad.

Investigación

Los proyectos de investigación son la base para lograr mayor capacidad de enfrentar diferentes problemas que se presentan en la producción como, por ejemplo: enfermedades, plagas, deficiencias de fertilización, riego, además de lograr obtener nuevos productos biológicos y liberar genotipos con cualidades de alto rendimiento. Actualmente, Ecuador sumado al esfuerzo de otros países latinoamericanos productores de cacao, deben ir paulatinamente encontrando soluciones para disminuir la contaminación por $\mathrm{Cd}$. A mediano plazo, los esfuerzos de investigación en este tema prioritario plantean algunas estrategias, entre ellas: seleccionar genotipos que tomen menor cantidad de $\mathrm{Cd}$, uso de enmiendas que permitan 
cambiar el contenido de materia orgánica, aumentar el pH y uso de microorganismos para el secuestro o retención de Cd del suelo (Meter, Atkinson, \& Laliberte, 2018)

Por otra parte, la alta variabilidad genética que posee Ecuador en el cacao es una ventaja que resalta y que abre puertas para seguir ofreciendo nuevos genotipos de mayor producción y tolerantes a varias enfermedades, tales como escoba de bruja y moniliasis. De hecho el INIAP recientemente liberó dos genotipos de alta producción y adaptado a varios pisos y regiones del Ecuador (Loor-Solórzano et al., 2019). Esto es un trabajo que implica tiempo e inversión en ensayos que validen la calidad del cultivar que se está seleccionando. Los institutos y centros de investigación y universidades tienen un papel protagónico en este campo y el estado debería establecer apoyos a estos centros de investigación para seguir fomentando investigaciones en función de lograr una cacao-cultura sustentable.

Se plantea la posibilidad de la creación de un centro nacional de investigación en cacao, pero valdría la pena evaluar la importancia que ha tenido las instituciones públicas como el INIAP y las universidades en el desarrollo de una investigación y tecnología nacional. Probablemente estas instituciones con una mayor inversión pueden promover y liderizar las líneas de investigación. Con la gran posibilidad de llegar a 500000 t de producción en un término de tres años y seguir creciendo, las bases de una tecnología nacional estarán basadas en la investigación.

\section{Conclusiones}

Un aspecto que resalta como inicio para lograr que Ecuador en el año 2025 llegue a 500000 t de producción, es consolidar entre los productores pequeños un buen sistema de cooperativas, con una organización orientada al apoyo al productor dándole el servicio y la formación técnica. Esto indudablemente mejorará la calidad del cacao y asegurará mejores precios al productor basado en precios diferenciales de calidad. A su vez urge el apoyo del estado ecuatoriano en mejorar en el sector rural los servicios básicos, en especial de salud, y comunicación. El rescate de la credibilidad de la buena calidad del cacao ecuatoriano a nivel internacional es prioritario y ello conlleva a implementar estrategias de mercadeo y rescate de clientes. La investigación sigue siendo fundamental para seguir dando soluciones a varios problemas e ir paulatinamente creando una tecnología nacional. Actualmente, una línea de investigación prioritaria es conseguir alternativas para tener almendras de cacao con menor cantidad de $\mathrm{Cd}$ y cultivares con mayor resistencia a las principales enfermedades tales como monilia y escoba de bruja. Es un trabajo colectivo nacional de los sectores que intervienen en la cadena de producción la concientización de establecer los cinco ejes de acción propuestos que permitirán una mayor resiliencia para poder aumentar la producción y mejorar el entorno y las condiciones en el que vive el agricultor. 


\section{Agradecimientos}

Los autores agradecen al MSc. Freddy Amores, MSc. Christian Marlín e Ingenieros Celso Averos y Reinaldo Ferreiras quienes generosamente han realizado la revisión del manuscrito y compartido sugerencias constructivas para mejorar el presente artículo.

\section{Contribución de los autores}

En concordancia con la taxonomía establecida internacionalmente para la asignación de créditos a autores de artículos científicos (https://casrai.org/credit/). Los autores declaran sus contribuciones en la siguiente matriz:

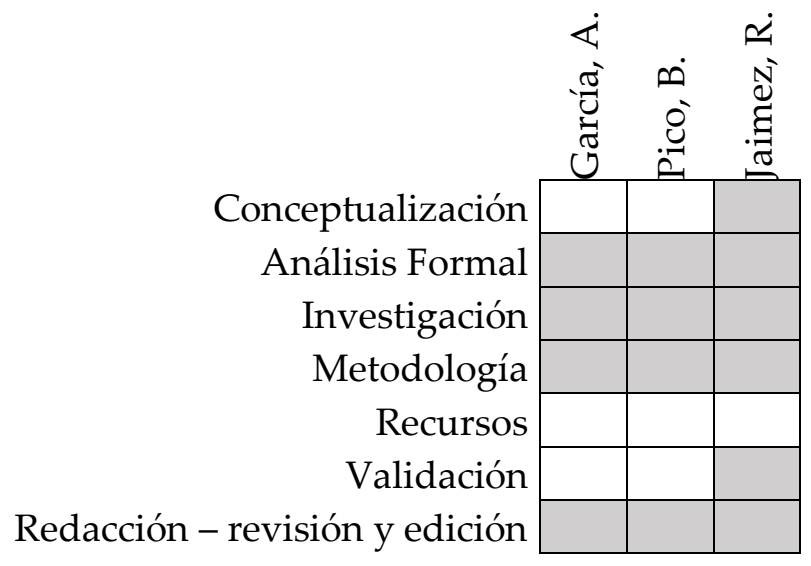

\section{Conflicto de Interés}

Los autores declaran que no existen conflictos de interés de ninguna naturaleza.

\section{Referencias}

ANECACAO (2019). Sector Exportador de Cacao. Recuperado de http://www.anecacao.com/uploads/estadistica/cacao-ecuador-2019.pdf .

ANECACAO (2020). Asociación Nacional de Exportadores de cacao - Ecuador. Recuperado de http://www.anecacao.com/index.php/es/revista.html.

ANECACAO (2021). Destino de las exportaciones de cacao en grano de Ecuador. Recuperado de http://www.anecacao.com/index.php/es/revista.html.

Argüello, D., Chavez, E., Lauryssen, F., Vanderschueren, R., Smolders, E., \& Montalvo, D. (2019). Soil properties and agronomic factors affecting cadmium concentrations in cacao beans: A nationwide survey in Ecuador. Science of the Total Environment Journal, 649,120127. https://doi.org/10.1016/j.scitotenv.2018.08.292.

Barraza, F., Moore, R. E. T., Rehkämper, M., Schreck, E., Lefeuvre, G., Kreissig, K., Coles, B. J., 
\& Maurice, L. (2019). Cadmium isotope fractionation in the soil - cacao systems of Ecuador: a pilot field study. RSC Advances, 9(58), 34011-34022. Recuperado de https://pubs.rsc.org/en/content/articlepdf/2019/ra/c9ra05516a

Barrera, V. H., Escudero, L., Racines, M. R., García, C., Arévalo, J., Casanova, T., ... Domínguez, J. (2019). La cadena de valor del cacao y el bienestar de los productores en la provincia de ManabíEcuador. (INIAP libro técnico No 171) Arco Iris Producciones, Quito, Ecuador. https://repositorio.iniap.gob.ec/bitstream/41000/5377/1/iniapsc383.pdf

Barrezueta-Unda, S., Blacio, W. M., \& Abad, C. Q. (2018). Análisis del cacao y café ecuatoriano desde su cadena de valor. Revista Científica Agroecosistemas, 6(3), 6-17. https://aes.ucf.edu.cu/index.php/aes/article/view/213.

Barrezueta-Unda, S., Prado, E., \& Sarmiento, R. J. (2017). Características del comercio de cacao a nivel intermediario en la provincia de El Oro-Ecuador. European Scientific Journal, 13(16),273. doi: 10.19044 / esj.2017.v13n16p273.

Braga, D. P. P., Domene, F., \& Gandara, F. B. (2019). Shade trees composition and diversity in cacao agroforestry systems of southern Pará, Brazilian Amazon. Agroforestry Systems, 93, 1409-1421 https://doi.org/10.1007/s10457-018-0250-6

Cadby, J., (2020). Vulnerabilities of the craft chocolate industry amidst the COVID-19 pandemic. Journal of Agriculture, Food Systems, and Community Development, 10(1), 219222. https://doi.org/10.5304/jafscd.2020.101.012

Callejas, D., Echevarría, J. M., Carrero, Y., Rodríguez-Morales, A. J., \& Moreira, R. (2020). The SARS-CoV-2 Pandemic in Latin America: the Need for Multidisciplinary Approaches. Current Tropical Medicine Reports, 7(4), 120-125. Recuperado de https://link.springer.com/content/pdf/10.1007/s40475-020-00219-w.pdf

Carr, M. K. V., \& Lockwood, G. (2011). The water relations and irrigation requirements of cocoa (Theobroma cacao L.): A review. Experimental agriculture, 47(4), 653-76. https://doi.org/10.1017/S0014479711000421 .

Carranza, M. S., Zapata, Y. P., Gallego, G., Rodriguéz, J. N., Carriel, J. M., Rosero, N. C., \& Muñoz, J. E. (2020). Genetic diversity of ecuadorian cocoa from the germplasm bank of tenguel-guayas Ecuador based in snp's, BIOAGRO, 32(2): 75-86. Recuperado de https://revistas.uclave.org/index.php/bioagro/article/view/2691/1677

CEPAL (2013). Diagnóstico de la Cadena Productiva del Cacao en el Ecuador. Secretaría Técnica del Comité Interinstitucional para el cambio de la matriz productiva - Vicepresidencia del Ecuador. Recuperado de https://www.vicepresidencia.gob.ec/wpcontent/uploads/2015/07/Resumen-Cadena-de-Cacao-rev.pdf

Coello, M. J., \& Haro, R. I. (2012). Caracterización de Sistemas Agroforestales Comúnmente Asociados al Cultivo de Cacao en la zona de Febres Cordero, provincia de Los Ríos (Tesis de grado presentada como requesito previo a la obyencion del titulo de Ingeniero 
Agronomo). Universidad Técnica Babahoyo, Babahoyo, Ecuador. http://dspace.utb.edu.ec/bitstream/handle/49000/956/T-UTB-FACIAG-AGR000162.pdf?sequence $=1 \&$ isAllowed $=\mathrm{y}$

Díaz-Montenegro, J., Varela, E., \& Gil, J. M. (2018). Livelihood strategies of cacao producers in Ecuador: Effects of national policies to support cacao farmers and specialty cacao landraces. Journal of Rural Studies 63:141-56. https://doi.org/10.1016/j.jrurstud.2018.08.004

Dreiss, M. L., \& Greenhil, E. S. (2008). Chocolate: Pathway to the gods. The sacred realm of chocolate in Mesoamerica. Tucson, Arizona: The University of Arizona Press.

Franzen, M., \& Borgerhoff, M. (2007). Ecological, economic and social perspectives on cocoa production worldwide. Biodiversity and conservation, 16(13), 3835-3849. Recuperado de https://link.springer.com/article/10.1007/s10531-007-9183-5

García, M., Montaño, L., \& Montoya, A. (2012). Comparative analysis of productive chain the production of cocoa between Colombia and Ecuador. Revista de Ciencias Agrícolas, 29(1), 99-112. Recuperado de https://revistas.udenar.edu.co/index.php/rfacia/article/view/372

Henry, C., Morillo, J. L., Salgado, V., \& Ulloa, W. (2018). Diagnostico y Benhmarking de la cadena de cacao. Consorcio AGRER-TYPSA Proyecto de asistencia Técnica para la reactivación productiva Post Terremoto. Manta: Ecuador

Ibarra, A. (2019). Análisis de la cadena de cacao en la provincia de los ríos Ecuador, Revista Observatorio de la Economía Latinoamericana En línea. https://www.eumed.net/rev/oel/2019/11/cadena-cacao-ecuador.html

ICCO (2021). Producción de granos de cacao. https://www.icco.org/about-us/international-cocoaagreements/doc_download/4577-production-qbcs-xlvi-no-2.html

INEC (2018). Documento metodológico de la Encuesta de Superficie y Producción Agropecuaria Continua (ESPAC). https://www.ecuadorencifras.gob.ec/documentos/webinec/Estadisticas_agropecuarias/espac/espac-2018/Metodologia de la operacion estadistica ESPAC 2018.pdf

INEC (2020). Documento metodológico de la Encuesta de Superficie y Producción Agropecuaria Continua (ESPAC). Recuperado de https://www.ecuadorencifras.gob.ec/documentos/webinec/Estadisticas_agropecuarias/espac/espac-2020/Metodologia\%20ESPAC\%202020.pdf

Jaimez, R. E., Araque, O., Guzman, D., Mora, A., Espinoza, W., \& Tezara, W. (2013). Agroforestry systems of timber species and cacao: survival and growth during the early stages. Journal of Agriculture and Rural Development in the Tropics and Subtropics, 114(1), 111. Recuperado de https://www.jarts.info/index.php/jarts/article/view/2012112642171

Loor-Solórzano, R. G., Amores-Puyutaxi, F. M., Vasco-Medina, S. A., Quiroz-Vera, J. G., 
Casanova-Mendoza, Garzón-Catota, A. I., ... Zambrano-Flores, F. G. (2019). INIAPEETP-800 Aroma Pichilingue, Nueva Variedad Ecuatoriana de Cacao Fino de alto Rendimiento. Revista Fitotecnia Mexicana, 42(2), 187-89. Recuperado de http://www.scielo.org.mx/scielo.php?script=sci_arttext\&pid=S0187-73802019000200187

Maddela, N. R., Kakarla, D., García, L. C., Chakraborty, S., Venkateswarlu, K., \& Megharaj, M. (2020). Cocoa-laden cadmium threatens human health and cacao economy: A critical view. Science of the Total Environment, 720. https://doi.org/10.1016/j.scitotenv.2020.137645

Márdero, S., Nickl, E., Schmook, B., Schneider, L., Rogan, J., Christman, Z., \& Lawrence, D. (2012). Sequías en el sur de la península de Yucatán: análisis de la variabilidad anual y estacional de la precipitación. Investigaciones Geograficas, (78),19-33. Recuperado de http://www.scielo.org.mx/scielo.php?script=sci_arttext\&pid=S0188-46112012000200003.

Medina, V., \& Laliberte. B. (2017). A review of research on the effects of drought and temperature stress and increased $\mathrm{CO}_{2}$ on Theobroma cacao L., and the role of genetic diversity to address climate change. Bioversity International: Costa Rica. Recuperado de https://cgspace.cgiar.org/handle/10568/89084

Mena-Coronel, K., \& Gutiérrez-Jaramillo, N. (2021). Efecto del Covid-19 y su incidencia financiera en las exportaciones del sector cacaotero. CIENCIA UNEMI 14(36); 34 - 44. https://doi.org/10.29076/issn.2528-7737vol14iss36.2021pp34-44p

Melo, C. J., \& Hollander, G. H. (2013). Unsustainable development: Alternative food networks and the Ecuadorian Federation of Cocoa Producers, 1995-2010. Journal of Rural Studies, 32(1), 251-63. https://agris.fao.org/agris-search/search.do?recordID=US201900109163

Meter, A., Atkinson, R. J., \& Laliberte, B. (2019). Cadmium in Cacao from Latin America and the Caribbean: A Review of Research and Potential Mitigation Solutions. Bioversity International: Costa Rica. Recuperado de https:/cgspace.cgiar.org/bitstream/handle/10568/102353/Bioversity-CAF-Review-CadCacao-13Nov2019-EN.pdf?sequence=9\&isAllowed=y

Morales, F. L., Carrillo, M. D., Ferreira, J. A., Peña. M. M., Briones, W. R., \& Albán, M. N. (2018). Cadena de comercialización del cacao nacional en la provincia de Los Ríos, Ecuador. Revista Ciencia y Tecnología, 11(1). https://dialnet.unirioja.es/servlet/articulo?codigo $=6550318$

Muller, M. W., \& Valle, R. R., (2012). Ecofisiologia do cultivo do cacaueiro. Ciencia Tecnologia e manejo de cacaueiro. MAPA: CEPLAC Brasil, 31-66.

Ortiz, J., Chungara, M., Ibieta, G., Alejo, I., Tejeda, L., Peralta, ... Peñarrieta, J. M., (2019. Determinación de teobromina, catequina, capacidad antioxidante total y contenido fenólico total en muestras representativasde cacao Amazónico Boliviano y su comparación antes y después del proceso de fermentación. Revista Boliviana de Química, $36(1), 40-50$. 
http://www.scielo.org.bo/scielo.php?pid=S0250-54602019000100004\&script=sci_arttext

Parada-Gutiérrez, O., \& Veloz-Cordero, R. (2021). Análisis socioeconómico de productores de cacao, localidad Guabito, provincia Los Ríos, Ecuador. Ciencias Holguín, 27(1), 1-18. http://www.ciencias.holguin.cu/index.php/cienciasholguin/article/download/1210/133.

Salgado, V. H., Morillo, J. L., \& Ulloa, W. (2018). Plan de mejora competitiva para el desarrollo agroindustrial de la cadena de cacao -chocolate. Consorcio AGRER-TYPSA Proyecto de asistencia Técnica para la reactivación productiva Post Terremoto. Manta: Ecuador.

Troya, M. B. (2013). Acción colectiva y cadenas de valor estudio de caso: Cadena de cacao y UNOCACE (Tesis de Maestria en Ciencias Sociales con Mencion en Desarrolllo Local y territorial). Tesis Inédita, Facultad Latinoamericana de Ciencias Sociales sede Ecuador (FLACSO), Ecuador. Recuperado de https://repositorio.flacsoandes.edu.ec/handle/10469/5851

Vasallao, M. (2017). Diferenciación y agregado de valor en la cadena ecuatoriana del cacao. REPIQUE. Revista de Ciencias Sociales, 1(1). Recuperado de http://revistasdigitales.utelvt.edu.ec/revista/index.php/repique/article/view/2

Vivanco-Saraguro, A. (2020). Teleducación en tiempos de COVID-19: brechas de desigualdad. CienciAmérica, 9(2). http://dx.doi.org/10.33210/ca.v9i2.307

Zarrillo, S., Gaikwad, N., Lanaud, C., Powis, T., Viot, C., Lesur, ... Valdez, F. (2018). The use and domestication of Theobroma cacao during the mid-Holocene in the upper Amazon, Nature Ecology and Evolution, 2(12), 1-10. https://doi.org/10.1038/s41559-018-0697-x 